\title{
IRREDUCIBLES IN THE LANDWEBER NOVIKOV ALGEBRA
}

\author{
ILAN KOZMA
}

\begin{abstract}
All the irreducible and reducible elements in the Landweber Novikov algebra are determined. A full set of relations mod reducibles is given.
\end{abstract}

1. Introduction. Let $S^{*}$ denote the Landweber Novikov algebra, and let $\bar{S}$ be the kernel of the augmentation map. The aim of this paper is to compute $Q\left(S^{*}\right)=\bar{S} / \bar{S}^{2}$, the module of irreducibles.

For every exponent sequence $\alpha$ with only finitely many nonzero terms, Landweber [1] and Novikov [2] define an operation $s_{\alpha} \in S^{*}$. Moreover, the $s_{\alpha}$ 's form a basis for $S^{*}$ as a $Z$-module.

For every exponent sequence $\alpha=\left(a_{1}, \ldots, a_{n}, \cdots\right)$, let $\|\alpha\|=\sum i a_{i}$, and $|\alpha|=\sum a_{i}$. Let $\Delta(a)$ denote the exponent sequence all of whose elements are zero except 1 in the $a$ th place. Our main theorem is

THEOREM 1.1. (a) $Q\left(S^{*}\right)$ is generated by $\left\{s_{p^{n} \Delta(1)}, s_{p^{n} \Delta(2)} \mid p\right.$ prime, $\left.n \geqslant 0\right\}$, with the only relations $p s_{p^{n} \Delta(1)} \in \bar{S}^{2}$ for $n \geqslant 2$ and every $p, p s_{p \Delta(1)} \in \bar{S}^{2}$ for $p \neq 2$, $p s_{p^{n} \Delta(2)} \in \bar{S}^{2}$ for $n \geqslant 1$ and $2\left(s_{\Delta(2)}+s_{2 \Delta(1)}\right) \in \bar{S}^{2}$.

(b) All the $s_{\alpha}$ 's are reducible except for $\alpha=p^{n} \Delta(1), p^{n} \Delta(2), 2 p^{n} \Delta(1)$. The only relations between irreducibles are those specified in (a) and $s_{p^{n} \Delta(2)}+$ $s_{2 p^{n} \Delta(1)} \in \bar{S}^{2}$ for $p \neq 2$ and $n>0$. [1].

Our main computational tool is the following theorem due to Landweber

Let $S_{*}$ be the dual algebra to $S^{*}$. Let $\sigma_{\alpha}$ be the dual basis to $s_{\alpha}$. Then $S_{*}$ is a polynomial algebra with generators $\left\{\sigma_{\Delta(a)}\right\}_{a \geqslant 1}$ and

THEOREM 1.2. The diagonal in $S_{*}$ is given by

$$
\phi_{*}\left(\sigma_{\Delta(a)}\right)=\sum_{\|\alpha\|+i=a}\left(\begin{array}{c}
i+1 \\
\alpha
\end{array}\right) \sigma_{\alpha} \otimes \sigma_{\Delta(i)} .
$$

ADDED IN PROOF. While writing this paper I heard that Aikawa [3] got the same results. I would like to thank Shibata for reading this paper and correcting many of the mistakes appearing in the original version.

2. Definition. If $\alpha=\left(a_{1}, \ldots, a_{n}, \ldots\right)$, let $\nu_{p}(\alpha)=\min _{i}\left\{\nu_{p}\left(a_{i}\right)\right\}$ and $\nu_{p}\left(n s_{\alpha}\right)$ $=\max \left\{0, \nu_{p}(\alpha)-\nu_{p}(n)\right\}$.

Received by the editors March 12, 1974 and, in revised form, October 9, 1974. AMS (MOS) subject classifications (1970). Primary 55B35, 55G25. 
Let us say that two exponent sequences $\alpha=\left(a_{1}, \ldots, a_{n}, \ldots\right), \beta$ $=\left(b_{1}, \ldots, b_{n}, \ldots\right)$ are disjoint if $a_{i} b_{i}=0$ for every $i$.

THEOREM 2.1. (a) For every $\alpha, \beta$ we have $s_{\alpha} \circ s_{\beta}=\lambda s_{\alpha+\beta}+\sum \lambda_{i} s_{\alpha_{i}}$ where $\left|\alpha_{i}\right|<|\alpha+\beta|$. If $\alpha$ and $\beta$ are disjoint, then $\lambda=1$. Moreover, $\nu_{p}\left(\lambda_{i} s_{\alpha_{i}}\right)$ $\leqslant \min \left\{\nu_{p}(\alpha), \nu_{p}(\beta)\right\}$.

(b) For $n>1$ there exists a $\lambda \in Z$ such that $\lambda s_{n \Delta(a)} \equiv \Sigma \lambda_{i} s_{\alpha_{i}} \bmod \bar{S}^{2}$, where $\left|\alpha_{i}\right|<n$ and $\nu_{q}\left(\lambda_{i} s_{\alpha_{i}}\right) \leqslant \nu_{q}(n)$ for every prime $q$. Moreover, $\lambda=1$ if $n$ is not a power of a prime, and $\lambda=p$ if $n=p^{k}$ for some prime $p$.

Proof of (a). We will prove (a) by passing to the dual. That is, if $\phi_{*}\left(\sigma_{\gamma}\right)=\lambda \sigma_{\alpha} \otimes \sigma_{\beta}+\cdots$ with $\lambda \neq 0$, then $|\gamma|<|\alpha+\beta|$ unless $\gamma=\alpha+\beta$. This will follow from 1.2 by trivial induction on $|\gamma|$. We also have to show that $\min \left\{\nu_{p}(\alpha), \nu_{p}(\beta)\right\} \geqslant \nu_{p}(\gamma)-\nu_{p}(\lambda)$. Let $r=\nu_{p}(\gamma)$, i.e. $\gamma=p^{r} \delta$. Hence,

$$
\phi_{*}\left(\sigma_{\gamma}\right)=\phi_{*}\left(\sigma_{\delta}\right)^{p^{r}}=\left(\sum \mu_{i} \sigma_{\alpha_{i}} \otimes \sigma_{\beta_{i}}\right)^{p^{\prime}},
$$

and we will get the results from the following lemma.

LEMMA 2.2. If $\left(\sum y_{i}\right)^{p^{r}}=\Sigma \lambda_{i} z_{i}$, where the $z_{i}$ 's are monomials in the $y_{i}$ 's, then $\nu_{p}\left(z_{i}\right)+\nu_{p}\left(\lambda_{i}\right) \geqslant r$, where $\nu_{p}(z)=\max \left\{t \mid \exists y\right.$ with $\left.z=y^{p^{t}}\right\}$.

Proof of 2.1 (b). From (a) we have that if $k+l=n$, then

$$
s_{k \Delta(a)} \circ s_{l \Delta(a)}=\left(\begin{array}{l}
n \\
k
\end{array}\right) s_{n \Delta(a)}+\sum \lambda_{i} s_{\alpha_{i}}
$$

where $\left|\alpha_{i}\right|<n$ and $\nu_{p}\left(\lambda_{i} s_{\alpha_{i}}\right) \leqslant \nu_{p}(n)$ for every prime $p$. But g.c.d. $\left\{\left(\begin{array}{c}n \\ k\end{array}\right)\right\}$ is the same $\lambda$ defined in the theorem, and, hence, we can take an appropriate linear combination of the above relations to get (b).

COROllary 2.3. For every $n$ and $\alpha$ we have that

$$
n s_{\alpha} \equiv \sum_{p, a, i} \lambda_{p, a, i} s_{p^{i} \Delta(a)} \bmod \bar{S}^{2}
$$

where $i \leqslant \nu_{p}\left(n s_{\alpha}\right)$.

Proof. The proof is by induction on $|\alpha|$. If $\alpha$ is not of the form $m \Delta(a)$, then there are disjoint $\beta, \gamma$ such that $\alpha=\beta+\gamma$. Then by $2.1(\mathrm{a}), n s_{\alpha} \equiv \sum n \lambda_{i} s_{\alpha_{i}}$ with $\nu_{p}\left(n \lambda_{i} s_{\alpha_{i}}\right) \leqslant \nu_{p}\left(n s_{\alpha}\right)$ and $\left|\alpha_{i}\right|<|\alpha|$. Apply now the induction hypothesis to $\alpha_{i}$ and $n \lambda_{i}$.

If $\alpha=m \Delta(a)$, do the same using 2.1(b).

LEMMA 2.4. For every $a \neq b$,

$$
\begin{aligned}
& \text { (a) } s_{\Delta(a)} \circ s_{\Delta(b)}=s_{\Delta(a)+\Delta(b)}+(b+1) s_{\Delta(a+b)} . \\
& \text { (b) } s_{\Delta(a)} \circ s_{2 \Delta(b)}=\lambda s_{\Delta(a)+2 \Delta(b)}+(b+1) s_{\Delta(b)+\Delta(a+b)} . \\
& \text { (c) } s_{2 \Delta(b)} \circ s_{\Delta(a)}=\lambda s_{\Delta(a)+2 \Delta(b)}+(a+1) s_{\Delta(b)+\Delta(a+b)}+\left(\begin{array}{c}
a+1 \\
2
\end{array}\right) s_{\Delta(a+2 b) .} .
\end{aligned}
$$

$\lambda$ is the same as in $b$ and $\lambda=1$ if $a \neq b$.

(d) $s_{\Delta(a)} \equiv 0 \bmod \bar{S}^{2}$ for every $a \neq 1,2$.

Proof. (a), (b), (c) are routine computations. To prove (d) we will have to separate cases: 
(1) $a$ odd, $a \neq 1$. Write $a=b+c$ with $b-c=1$. Then by (a), $\left[s_{\Delta(b)}\right.$, $\left.s_{\Delta(c)}\right]=(b-c) s_{\Delta(a)}=s_{\Delta(a)}$.

(2) $a$ even, $a \neq 2$. Write $a=b+c$ with $b-c=2$. Then as in (1) we get $2 s_{\Delta(a)} \equiv 0 \bmod \bar{S}^{2}$.

Let $a=2+2 b$. Using (b), (c) and (a) we get that

$$
\left[s_{\Delta(2)}, s_{2 \Delta(b)}\right]=(b-2) s_{\Delta(b)+\Delta(b+2)}+3 s_{\Delta(a)}
$$

and

$$
s_{\Delta(b+2)} \circ s_{\Delta(b)}=s_{\Delta(b)+\Delta(b+2)}+(b+1) s_{\Delta(a)} .
$$

Combining both we get

$$
s_{\Delta(a)} \equiv[(b+1)(b-2)-2] s_{\Delta(a)} \bmod \bar{S}^{2} .
$$

But $(b+1) \cdot(b-2)$ is even and, hence, $s_{\Delta(a)} \in \bar{S}^{2}$.

LEMMA 2.5.

$$
\begin{aligned}
s_{p^{n} \Delta(a)} \circ s_{p^{n} \Delta(b)} \equiv & (b+1)^{p^{n}} s_{p^{n} \Delta(a+b)}+s_{p^{n}(\Delta(a)+\Delta(b))} \\
& +\sum_{c ; k<n} \lambda_{c, k} s_{p^{k} \Delta(c)} \bmod \bar{S}^{2} \text { for } a \neq b . \\
s_{p^{n} \Delta(a)} \circ s_{2 p^{n} \Delta(b)} \equiv & \lambda^{p^{n}} S_{p^{n}(\Delta(a)+2 \Delta(b))}+(b+1)^{p^{n}} s_{p^{n}(\Delta(b)+\Delta(a+b))}
\end{aligned}
$$

$$
+\sum_{c ; k<n} \lambda_{c, k} s_{p^{k} \Delta(c)} \bmod \bar{S}^{2} \text {. }
$$

$$
s_{2 p^{n} \Delta(b)} \circ s_{p^{n} \Delta(a)} \equiv \lambda^{p^{n}} S_{p^{n}(\Delta(a)+2 \Delta(b))}+(a+1)^{p^{n}} s_{p^{n}(\Delta(b)+\Delta(a+b))}
$$

$$
+\left(\begin{array}{c}
a+1 \\
2
\end{array}\right)^{p^{n}} s_{p^{n} \Delta(a+2 b)}+\sum_{c ; k<n} \lambda_{c, k} s_{p^{k} \Delta(c)} \bmod \bar{S}^{2} .
$$

The constant $\lambda$ appearing in (b) and (c) is the same $\lambda$ as in 2.4 .

(d) For every $a \neq 1,2$ we have $s_{p^{n} \Delta(a)} \in \bar{S}^{2}$.

Proof. The proof of (a), (b) and (c) are identical, so we will prove (b). By 2.1 we have that

$$
s_{p^{n} \Delta(a)} \circ s_{2 p^{n} \Delta(b)}=\lambda^{p^{n}} S_{p^{n}(\Delta(a)+2 \Delta(b))}+\sum \lambda_{i} s_{\alpha_{i}}
$$

with $\left|\alpha_{i}\right|<3 p^{n}$, and that for every prime $q \neq p, \quad \nu_{q}\left(\lambda_{i} s_{\alpha_{i}}\right)=0$. We want to show that the only possible $\alpha_{i}$ in the sum with $\nu_{p}\left(\alpha_{i}\right) \geqslant n$ is $p^{n}(\Delta(b)+\Delta(a+b))$. This will imply (b) by Corollary 2.3 .

But if $\alpha_{i}=p^{n} \beta$ and $\left|\alpha_{i}\right| \leqslant 2 p^{n}$, then $\beta$ must be of the form $\Delta(t)$ or $\Delta(t)+\Delta(s)$ or $2 \Delta(t)$. An immediate check leaves the only possibility $\beta$ $=\Delta(a)+\Delta(a+b)$.

(d) The proof is by induction on $n$, the case $n=0$ having been done in $2.4(\mathrm{~d})$. For $n>0$ one follows the proof of 2.4(d). The only extra fact which is needed is that $p s_{p^{n} \Delta(a)} \in \bar{S}^{2}$ for $a \geqslant 2$, but this will follow from $p s_{p^{n} \Delta(a)}$ ${ }_{43} \equiv \Sigma_{i<n ; b} \lambda_{i, b} s_{p^{i} \Delta(b)}$ and our induction hypothesis. (Note that in the above sum we have $b>2$, so induction applies.)

Proof of Theorem 1.1. Let $\|\alpha\|=n$ with $n$ not of the form $p^{m}$ or $2 p^{m}$. Then $s_{\alpha} \equiv \Sigma_{p, i, a} \lambda_{p, i, a} s_{p^{i} \Delta(a)} \bmod \bar{S}^{2}$ where $a \neq 1,2$. Then by $2.5(\mathrm{~d}), s_{\alpha}$ is reducible. 
If $\|\alpha\|=p^{n}$ or $2 p^{n}$, but $\alpha \neq p^{n} \Delta(1), p^{n} \Delta(2)$ or $2 p^{n} \Delta(1)$, then $\nu_{p}(\alpha)<n$ and $s_{\alpha} \equiv \Sigma_{i, a} \lambda_{i, a} s_{p^{i} \Delta(a)}$ with $a \neq 1,2$ and, as before, $s_{\alpha}$ is reducible.

As for the remaining cases, we have already shown in the proof of $2.5(\mathrm{~d})$ that $p s_{p^{n} \Delta(a)} \in \bar{S}^{2}$ for $a \geqslant 2$. The same proof works if $a=1$ and $p>2$. To show that these are the only relations, look at:

$\phi_{*}\left(\sigma_{p^{n} \Delta(1)}\right)=\left(\sigma_{\Delta(1)} \otimes 1+1 \otimes \sigma_{\Delta(1)}\right)^{p^{n}}$. So in any relation where $s_{p^{n} \Delta(1)}$ appears, it is with coefficient divisible by $p$. Hence, it is irreducible.

$\phi_{*}\left(\sigma_{p^{n} \Delta(2)}\right)=\left(\sigma_{2} \otimes 1+2 \sigma_{1} \otimes \sigma_{1}+1 \otimes \sigma_{2}\right)^{p^{n}}$. Hence, the only relation in which $s_{p^{n} \Delta(2)}$ appears with a coefficient which is not divisible by $p$ is

$$
s_{p^{n} \Delta(1)} \circ s_{p^{n} \Delta(1)}=2^{p^{n}} s_{p^{n} \Delta(2)}+\left(\begin{array}{c}
2 p^{n} \\
p^{n}
\end{array}\right) s_{2 p^{n} \Delta(1)}+\cdots .
$$

Similarly, the previous relation is the only interesting one for $s_{2 p^{n} \Delta(1)}$. The other terms in this expression are all in $\bar{S}^{2}$.

If $p>2$ we have

$$
\left(\begin{array}{c}
2 p^{n} \\
p^{n}
\end{array}\right) \equiv 2^{p^{n}} \equiv 2 \quad \bmod p .
$$

Hence, $2\left(s_{2 p^{n} \Delta(1)}+s_{2 p^{n} \Delta(2)}\right) \in \bar{S}^{2}$. We also have $p\left(s_{2 p^{n} \Delta(1)}+s_{p^{n} \Delta(2)}\right) \in \bar{S}^{2}$ and we get our theorem for $p \neq 2$. If $p=2$ we have

$$
\left(\begin{array}{c}
2 p^{n} \\
p^{n}
\end{array}\right) \equiv 2 \bmod 4
$$

Hence, $2 s_{2^{n+1} \Delta(1)} \in \bar{S}^{2}$ for every $n \geqslant 1$, which finishes our proof.

\section{REFERENCES}

1. P. S. Landweber, Cobordism operations and Hopf algebras, Trans. Amer. Math. Soc. 129(1967), 94-110. MR36 \#2145.

2. S. P. Novikov, The method of algebraic topology from the viewpoint of cobordism theories, Izv. Akad. Nauk SSSR Ser. Mat. 31(1967), 855-951 = Math. USSR Izv. 1(1967), 827-922. MR36 \#4561.

3. Shibata Katsuyuki, T. Aikawa's theorem on Landweber Novikov algebra of complex cobordism theory (preprint).

Department of Theoretical Mathematics, Weizmann Institute of Science, Rehovot, ISRAEL 